\title{
Face Recognition for E-Attendance for Student and Staff
}

\author{
${ }^{1}$ Anusaya Tantak, ${ }^{2}$ Archana Sudrik, ${ }^{3}$ Archana Kale, ${ }^{4}$ Rutuja Mehetre, \\ ${ }^{5}$ Prof.Ms.S.S. Pophale \\ ${ }^{1,2,3,4}$ Information Technology, Dr. Vithalrao Vikhe Patil College of Engineering, Ahmednagar, Maharashtra, \\ ${ }^{5}$ Asst.Prof.,Information Technology, Dr. Vithalrao Vikhe Patil College of Engineering, Ahmednagar, \\ Maharashtra, India.
}

\begin{abstract}
The issue of taking attendance each and every period by the teacher is very time consuming and tiring as well Attendance management of student in any institution is a very lengthy process and even time consuming. Furthermore, biometrics attendance system is also available. These methods too are time consuming, since each time student has to form a queue for scanning their thumb using real time camera. The Attendance will be recorded by using camera, which will capture images of students and staff. It will then compare the faces with the Student's and Staff database and marks the attendance. Such automated systems will help largely reduce the manual labor and discrepancies otherwise involved in attendance maintenance.
\end{abstract}

Keywords: Face Detection, Face Recognition, Attendance System

\section{Introduction}

Nowadays, taking attendance in any field is very important task so as to maintained the record of student, employee etc. Traditionally, student attendance is taken manually by using attendance sheet given by faculty member in class which is very time consuming. We observed that the technique used was very time consuming and even many demerits has been observed by us such as wastage of paper, interruption in classroom etc. previously a system has been proposed by continues observation which improves the performance for the estimation of the attendance. Implementation of the system has to be carried out on accordance of some techniques named as face detection and face recognition. The Face detection and face recognition are very advanced in terms of computer authentication technology [1]. The technology of student attendance system is used to support the teacher for checking student attendance in modern way. The system is going to work by some techniques such as the image is captured by web camera of laptop is then processed towards the detection as the detected face image is obtained face recognition has to be done which is divided into further parts namely face alignment, preprocessing, feature extraction, face matching where the image is converted into gray scale image and the result has to be seen[1][2]. This has been done by using PCA algorithm. This technique is considered to be one of the most successful for image processing or analysis.

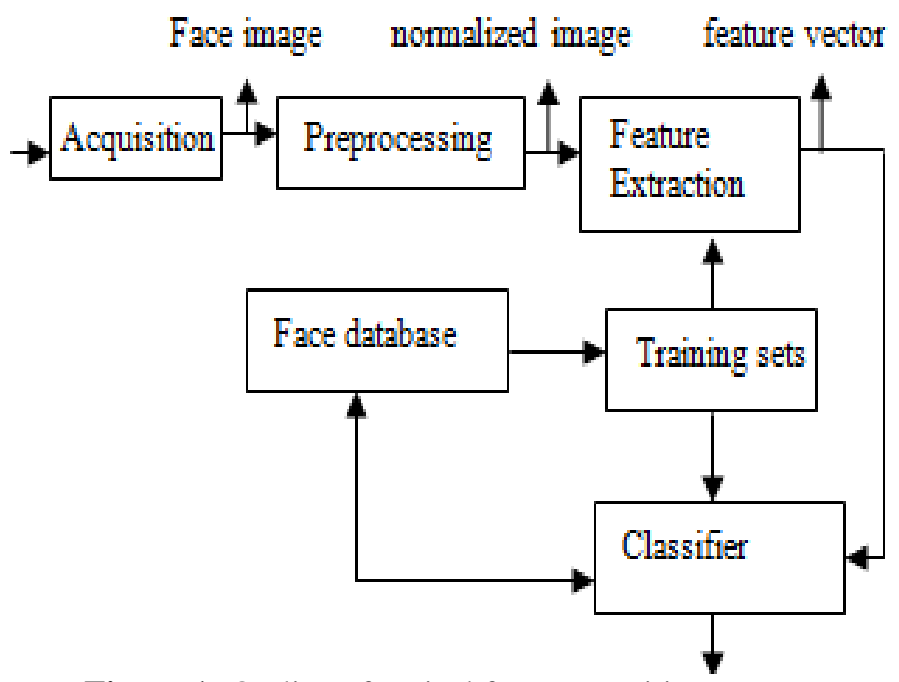

Figure 1: Outline of typical face recognition system

Acquisition Module: It is the module where the face under consideration is presented to the system. The user prepares to present a face image to face recognition module. It can request a face image from several different environments: the face can be image file from files or captured by video or scanned from paper[2]. 
Pre-Processing Module: It is face normalized and desires, they are enhanced to improve the performance of recognition system.

Feature Extraction Module: After performing some pre-processing (if-necessary), the normalized face image is presented to the feature extraction module in order to find the key features that are going to be used for classification. In other words, this module is responsible for composing a feature vector that is well enough to represent the face image.

Classification Module: In this module, with the help of pattern classifier, extracted features of the face image is compared with the ones stored in a face library or face database. After doing this comparison, face image is classified as either known or unknown.

Training Set: Training sets are used during the "learning phase" of the face recognition process in supervised face classifiers. The feature extraction and the classification modules make direct use of the face library.

\section{Literature Survey}

Here the details about the referred paper, author are given below:

In "Identification of human faces" by A.J. Gold Stein, L. D. Harmon, and A.B. Lesk used 21 different facial marks such as hair color and lip thickness for processing the image recognition. The problem was, it had to be manually computed the measurements and location. How well can human faces are identified by humans and by computers, using subjectively judged "feature" descriptions like long ears, wide-set eyes, etc.? Three classes of experiments are reported: 1) Gathering, analysis, and assessment of face-feature data for 255 faces. 2) Computer identification-studies. 3) Human identification-studies. A set of 22 features was evolved from an initially larger set to provide relevant, distinctive, relatively independent measures which can be judged reliably. Computer studies and a mathematical model established limits of performance of a person attempting to isolate a face from a population using feature descriptions. The model predicts that under certain conditions approximately 6 of an individual's features are required to isolate him from a population of 255. Human experiments under similar conditions showed unique identification occurred with an average of about 7 features. The model predicts that for a population of $4 \times 10^{6}$, only 14 feature-descriptions are required. These studies form a foundation for continuing research on real-time man-machine interaction for computer classification and identification of multidimensional vectors specified by noisy components[3].

In "Face Recognition Based on Principal Component Analysis" by Ali Javed's paper was a research work of face recognition system by using PCA which is eigenvector based multivariate analyses. The purpose of the proposed research work is to develop a computer system that can recognize a person by comparing the characteristics of face to those of known individuals. The main focus is on frontal two dimensional images that are taken in a controlled environment i.e. the illumination and the background will be constant. All the other methods of person's identification and verification like iris scan or finger print scan require high quality and costly equipment's but in face recognition we only require a normal camera giving us a 2-D frontal image of the person that will be used for the process of the person's recognition. Principal Component Analysis technique has been used in the proposed system of face recognition. The purpose is to compare the results of the technique under the different conditions and to find the most efficient approach for developing a facial recognition system[4]

In "Face Recognition Based Automated Attendance Management System using Principal Component Analysis" by Aalam Gumber and Navneet Kaur gave face Recognition based Automatic Attendance Management System using Principle Component Analysis is proposed. The system consists of a database of a set of facial patterns for each individual. The characteristic features called 'eigen faces' are extracted from the stored images using which the system is trained for subsequent recognition of new images[5].

\section{Proposed System}

In the earlier systems the attendance of the students has been marked for the whole day at once. Thus if a student is present for only a lecture and then left the class, even then he will be marked as present for whole day. We propose a system for automated attendance of the student which will mark the attendance for each lecture in the classroom via face recognition. We will create the database of face of each of the student. Image will be Capture with the help of Web Camera of Laptop[6]. The image of student will be considered for further processing. Then the detected face images are been compared with the images of the student in the database for recognition process. The database contains the record of all of the students in each class. If image matched with database image then student with that face is marked as present. The whole student attendance record will be maintained by Mail Server[6][7]. The Attendance will be calculated daily and Monthly and whole report will send to student on mail account. 


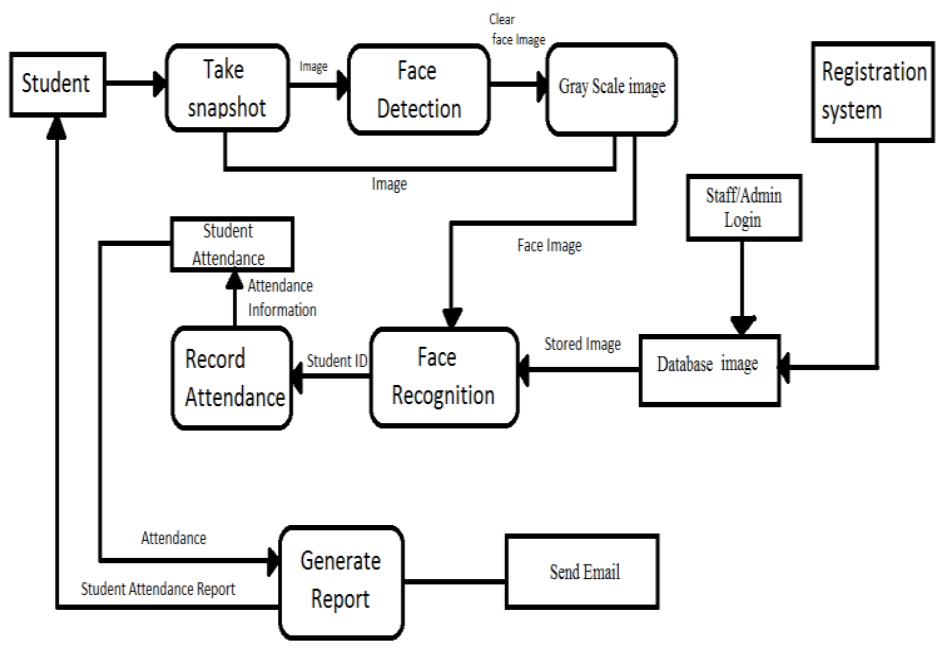

Figure 2 : System Architecture

Face detection has been applied on the images after preprocessing. Face detection is a starting point for face recognition. The system performs the face detection of all images taken by the digital camera. In database phase, the proposed system creates a database and stores all the preprocessed images in this database and finally system creates a database matrix and the system perform all the operations on the database matrix. The Principle Component Analysis technique has been applied on the images of the database system. The proposed system uses the test images stored in the database [8]. The system implement Principal Component Analysis algorithm on the test images. Now system compares the capture images taken by digital camera and test images one by one. Finally the system provides the results which show the name of the person and face marked by the bounding box[8][9]. The system flow chart is shown in Fig.2.

Face Detection: Face detection is a technology that determines the location and sizes of human faces in an image. It detects faces and ignores anything else, such as building, chairs, and trees. It is a starting point for face recognition. Most of the face detection methods focus on detecting frontal faces. These methods are categorized into four types: Knowledgebase, Feature invariant, Template matching and Appearance-Based. Each method involves color segmentations, pattern matching, statistical analysis and complex transform. Face detection is an important part of face recognition as to implement the automatic face recognition[10].

Face Recognition: Face Recognition is automatic identification or verification of a person from an image/video. It is one of the most active and widely used techniques because of its reliability, accuracy in the process of recognizing and verifying the person's identity. Problem that may occur with face recognition are different people may look similar; characteristic of the face may change with time. Face can be recognized by two approaches that are based on geometry of face and based on appearance of face. The recognition process is done by comparing the extracted features from the image with the one previously stored in the database. Face recognition can be implemented bay using Principal Component Analysis (PCA), Linear Discriminant Analysis (LDA) and Discrete Cosine Transform (DCT) etc[11].

\section{Algorithm Implementation}

Principal Component Analysis (PCA)

PCA is a statistical method under the broad title of factor analysis. The purpose of PCA is to reduce the large dimensionality of the image space (observed variables) to the smaller intrinsic dimensionality of feature space (independent variables), which are needed to describe the image data economically. In this case, the observed variables in image space are strongly correlated and variables in smaller feature space are uncorrelated. It is well known that there exist significant statistical redundancies in natural images. The purpose is to reduce the dimensionality of a data set (sample) by finding a new set of variables, smaller than the original set of variables that nonetheless retains most of the sample's information i.e. variations present in the sample, given by the correlations between the original variables[11][12]. The new variables, called Principal Components (PCs), are uncorrelated, and are ordered by the fraction of the total information each retains[12][13]. 


\section{Steps of PCA}

1. Let us consider a set of $\mathrm{N}$ sample images $(X 1, X 2, \ldots X N)$ taking values in n-dimensional image space, and assume that each image belongs to one of classes $(C 1, C 2, \ldots C c)$. Let $N i$ be the number of samples in class $C i(\mathrm{i}=1,2, \ldots \ldots \mathrm{c}), \mu i-1 \mathrm{Nix \in CiX}$ be the mean of the samples in class $\mathrm{Ci}, \mu=1 \mathrm{NxiNi=1}$ be the mean of all samples.

2. A linear transformation maps the original $n$-dimensional image space into an $m$-dimensional feature space, where $\mathrm{m}<\mathrm{n}$. The new feature vectors $y k \in \mathbb{R} m$ are defined by the following linear transformation: $Y k=$ WTxk (1.1) Where $k=1,2, \ldots ., \mathrm{N}$ and $W \in \mathbb{R} n \times m$ is a matrix with orthonormal columns.

3. Total scatter matrix $S T$ (or covariance matrix) is defined as [13]: $S T=1 N x k-\mu(x k-\mu) T N k=1=1 N A A T$ (1.2) After applying the linear transformation $W T$, the scatter of the transformed feature vectors $\{y 1, y 2 \ldots$ $, y N\}$ is WTSTW. In PCA, the projection Wopt is chosen to maximize the determinant of the total scatter matrix of the projected sample, i.e. $W o p t=\operatorname{argmax} w W T S T W=[w 1, w 2 \ldots, w m]$

Where $\{w i \mid i=1,2, \ldots m\}$ is set of $\mathrm{n}$-dimensional eigenvectors of $S T$ corresponding to the $m$ largest eigen values $\{\lambda i \mid i=1,2, \ldots, m\}$, i.e. $S T w i=\lambda i w i i=1,2, \ldots, \mathrm{m}(1.4)$ The steps involved in implementing the algorithm are [13-16]: Project training database images to feature space:

4. A set of images which is made up of classes of images of images of subjects that should be recognized by the system is used as training set. Represents the $2 \mathrm{D}$ images $\{x 1, x 2, \ldots, x N\}$ in the database in terms of 1D column vector $X$ as given below: $X=\{x 1, x 2, \ldots, x N\}$ The average training set image $(\mu)$ is defined by: $\mu=1 N x i N I=1$ Each trainee image differs from the average image by vector ( ) $\phi i=x i-\mu(1.7)$ Total scatter matrix or covariance matrix is calculated from $\phi$ as follows : $C=1 N \phi i N i=1 \phi i T=A A T$ (1.8) $A A T$, where $A=[\Phi 1, \Phi 2, \Phi 3, \ldots, \Phi N]$ (1.9) Calculate the eigen values $\lambda k$ and eigenvectors $w k$ of the covariance matrix $\mathrm{C}$.

\section{Implementation Result}

Images taken in the database are gray scale. A colored face image if given as input test image is to be first converted to gray scale image as gray scale images are easier for applying computational techniques in image processing. Matlab 2013a is used for coding. A gray scale face image is scaled for a particular pixel size as $243 \times 320$ because many input images can be of different size whenever we take an input face for recognition. All the images in our database are of same size i.e. $243 \times 320$. A test image for recognition is tested by comparing to the stored data set. Threshold value of the test face image to eigenface space which is Euclidean distance is taken as 1 which classifies the face as known or unknown. Six different images for different mentioned condition were taken to test for different people. We can observe that normal expressions are recognized as face space efficiently because facial features are not changed much in that case and in other cases where facial features are changed efficiency is reduced in recognition. After reading the database and the test image, the first step in automatic attendance system is to ask user if he wants to apply any preprocessing to the test image. The figures show the snapshots of Matlab window for the same. Preprocessing is required if the test image is noised. Noise may affect the image due to improper lighting conditions other factors. In this dissertation we consider it to be salt and pepper noise. If the user chooses to apply preprocessing and presses 1, noise is first added to test image, then removed by using median filtering. The noised image and the preprocessed image are shown in figure 4 and 5 respectively. On the other hand, if the user presses 2 and opts not to apply preprocessing on test image, the system bypass the filtering module and straight away moves to the recognition module.

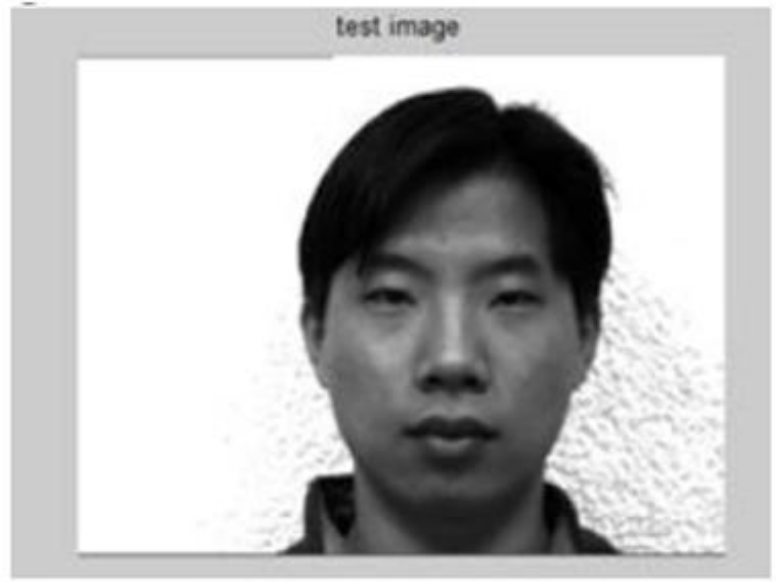

Figure 3: Test Image 

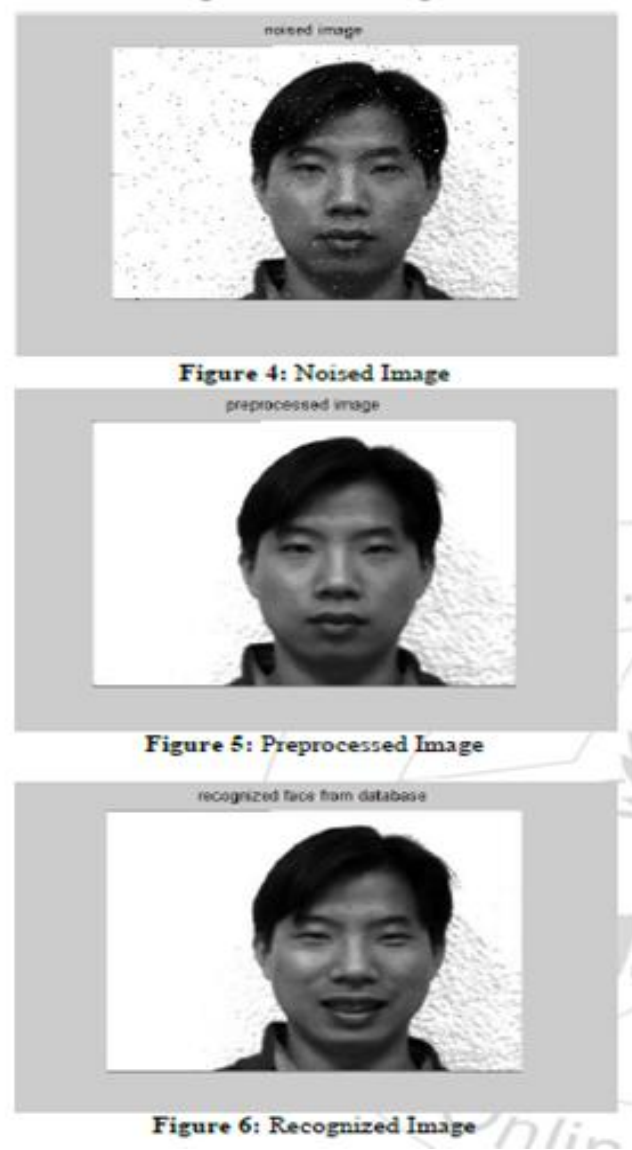

In the recognition module, the test image is compared with the images present in database. If a positive match is found, the recognized face with respect to the test image in figure 6. After recognizing the image, the roll number of recognized person is displayed on the command window. The attendance sheet for the current iteration is updated in an excel sheet. Snapshot of this process is shown in figure 7. Excel sheet of recognized person is shown is shown in figure 8 .

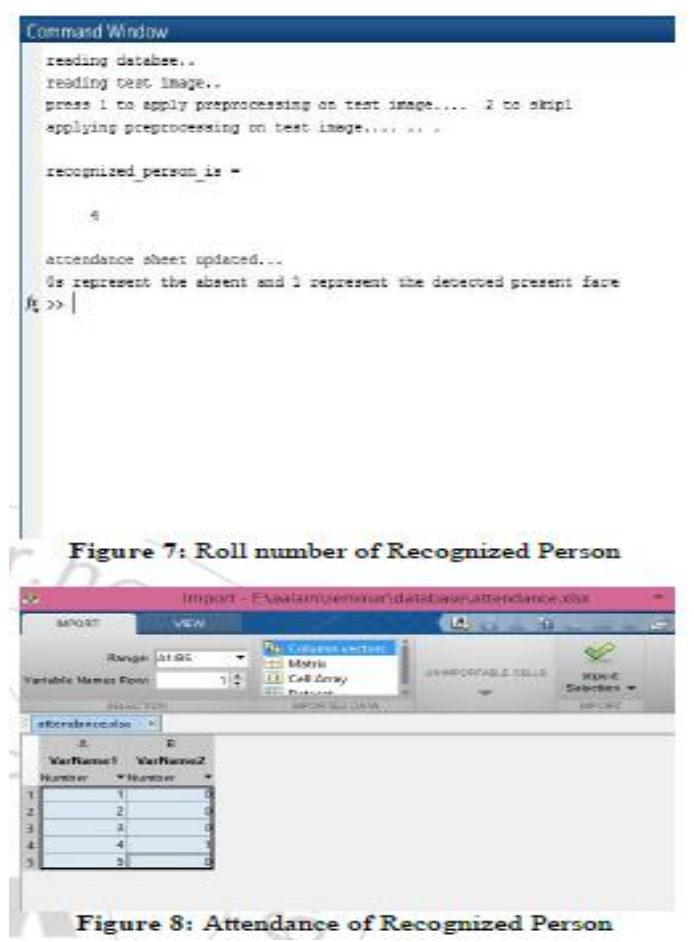




\section{Conclusion}

In this paper, we implemented the face recognition system using Principal Component Analysis and Eigen face approach. The system successfully recognized the human faces and worked well with different facial expressions and addition of salt and pepper noise. The face recognition system was used to create an automatic attendance updating system based on face recognition. This attendance system can be used to take attendance of different individuals on the basis of their face images. The system estimates the attendance of each student by capturing image by web camera of laptop. Then image will go for further processing. The system allows the lecturer to check his/her student attendance automatically by using personal computer (PC) without any extra cost and effort.The system automatically updates the attendance of the individuals and mark present/absent for them in an excel sheet. The excel sheet used to update attendance is overwritten every time the program is executed. Hence the user has to save the current attendance in the memory in order to use it for future reference. From the results performed on the database, it is evident that the attendance system based on face recognition performs satisfactorily. Hence it can be concluded that the present algorithm demonstrates better performance with respect to speed, low false positive rate and high accuracy.

\section{Acknowledgements}

It is our advantage to acknowledge with deep sense of gratitude to our project guide Prof. Ms. S.S. Pophale and Prof. Ms. M.S. Gunjal whose supervision, inspiration and valuable discussion has helped us to complete our project. Their guidance proved to be the most valuable to overcome all the complications in the fulfillment of this project on "Result Analysis from PDF File". We are thankful to Principal Dr. Jayakumar Jayaraman or direct or indirect help in the completion of this project. Last but not least, this acknowledgement would be incomplete without rendering our sincere gratitude to all those who have helped us in the completion of this project.

\section{References}

[1]. Vidhate, Deepak A and Kulkarni, Parag "Performance enhancement of cooperative learning algorithms by improved decision making for context based application", International Conference on Automatic Control and Dynamic Optimization Techniques (ICACDOT)IEEE Xplorer, pp 246-252, 2016

[2]. Abhishek Jha, "Classroom Attendance System Using Face Recognition System", The International journal of Mathematics, science, technology and Management, Vol. 2, ISSD: 2319-8125.

[3]. Vidhate, Deepak A and Kulkarni, Parag "Innovative Approach Towards Cooperation Models for Multi-agent Reinforcement Learning (CMMARL)", Springer Nature series of Communications in Computer and Information Science, Vol. 628,pp. 468478,2016

[4]. Ali Javed "Face Recognition Based on Principal Component Analysis" International Journal of Image, Graphics and Signal Processing, Vol. 2, pp.38-44, 2013

[5]. Roshan Tharanga, S. Samarakoon, "Smart attendance using real time face recognition," IEEE, 2013.

[6]. Vidhate, Deepak, A; Kulkarni, Parag "Multilevel Relationship Algorithm for Association Rule Mining used for Cooperative Learning" in International Journal of Computer Applications, 86(4), pp.20-27, 2014

[7]. Balwant Singh, Sunil Kumar, Paurush Bhulania, "Lecture Attendance System WITH Face Recognition And Image Processing", International Journal Of Advance Research In Science And Engineering, Vol. No.2, Issue No.3, March, 2013 ISSN-2319-8354(E)

[8]. Pallabi Saikia, Margaret Kathing, “A Biometric Authentication Approach using Face Recognition System”, International Journal of Advanced Research in Computer Science and Software Engineering, March 2014, Vol. 4, Issue 3

[9]. Vidhate, Deepak A and Kulkarni, Parag "Multi-agent Cooperation Methods by Reinforcement Learning (MCMRL)", Elsevier International Conference on Advanced Material Technologies (ICAMT)-2016\}No. SS-LTMLBDA-06-05,2016

[10]. Aalam Gumber , Navneet Kaur "Face Recognition Based Automated Attendance Management System using Principal Component Analysis" International Journal of Science and Research (IJSR), Volume 4 Issue 6, pp 971-975, June 2015

[11]. W. Zhao, R. Chellappa, P. J. Phillips and A. Rosenfeld, "Face recognition: A literature survey,” ACM Computing Surveys, 2003, vol. 35, no. 4, pp. 399-458.

[12]. Vidhate, Deepak, A; Kulkarni, Parag (2013) : "Mining Association Rule by Multilevel Relationship Algorithm: An Innovative Approach for Cooperative Learning" in International Journal of Emerging Trends \& Technology in Computer Science (IJETTCS), 2(6), pp. 130-137

[13]. Vidhate, Deepak, A; Kulkarni, Parag (2012) : "Review on Context Based Cooperative Machine Learning with Dynamic Decision Making in Diagnostic Applications" in International Conference on Computing, Communication and Information Technology (ICCCIT 2012) 1(1), pp. 161-166 